\title{
Der Un-Sprechakt des Jahres. Zum Problem der Parteilichkeit in linguistischer Sprachkritik
}

\author{
Ralf Vogel
}

\author{
11.11 .14
}

\section{Einführung}

Unter dem Titel „Alternativen zum Elfenbeinturm. Die Linguistik will stärker in die Öffentlichkeit hineinwirken" veröffentlichten die TeilnehmerInnen einer Tagung an der TH Aachen eine „Aachener Erklärung zur Rolle der Sprachwissenschaft in der Gesellschaft" (Bär/Niehr, 2012) in der vor allem dazu aufgerufen wird, dass SprachwissenschaftlerInnen sich mit ihren Positionen und Inhalten stärker in der Öffentlichkeit Gehör verschaffen sollten. In diesem Beitrag möchte ich - in kritischer Unterstützung der Aachener Erklärung - auf das Problem von Parteilichkeiten und Parteinahmen in sprachkritischen Stellungnahmen aufmerksam machen. Ich werde insbesondere die Aktion „Unwort des Jahres“ genauer unter die Lupe nehmen, weil sie die sprachkritische Wortmeldung von LinguistInnen mit der weitaus größten Resonanz ist.

Es geht mir darum, das antidemokratische Potential öffentlicher Sprachkritik aufzuzeigen und daraus Schlussfolgerungen für linguistische Sprachkritik abzuleiten. Dieses Potential begründet sich wie folgt:

1. Sprachgebrauch ist sprachliches Handeln durch konkrete Personen und grundsätzlich nicht von ihnen zu trennen. Die Kritik an einem Sprachgebrauch ist notwendigerweise immer zugleich Kritik an den so sprachlich Handelnden und somit parteilich.

2. Die Äußerung von Sprachkritik ist selbst Sprachhandeln durch die SprachkritikerInnen. Da Sprachkritik Kritik an Personen ist, kann sie auch diskriminierend wirken, und somit den eigenen Wertmaßstäben zuwiderlaufen.

3. Jedes soziale Handeln geschieht notwendig aus einer subjektiven Perspektive heraus. Im Falle der Sprachkritik gilt dies für Kritiker und Kritisierte gleichermaßen. Sprachkritik, die bloß kritisch auf eine von der eigenen abweichende Perspektive reagiert, käme dem undemokratischen Versuch gleich, eine andere Meinung zu unterdrücken.

Sprachkritik, wie sie heute zumeist verstanden wird, ist Kritik des Sprachgebrauchs. Hierbei unterscheide ich Formkritik von Angemessenheitskritik. Formkritik bezieht sich auf Sprachhandlungen, in denen die vermeintlich falsche Form verwendet wird, beispielsweise die vermeintlich falsche grammatische Konstruktion oder die vermeintlich falsche Bedeutung für ein Lexem. Angemessenheitskritik bezieht sich auf Sprachhandlungen, in denen sich auf vermeintlich unangemessene, unverständliche oder verwerfliche Weise ausgedrückt wird.

Beiden Typen von Sprachkritik liegen bestimmte Beurteilungskriterien zugrunde, die freilich nicht immer konsequent expliziert werden. Die Stichhaltigkeit einer Kritik hängt allerdings wesentlich von der Stichhaltigkeit dieser Kriterien ab. Ein typisches 
Muster sprachwissenschaftlicher Repliken auf laienlinguistische Sprachkritik besteht konsequenterweise darin, dass deren Maßstäbe und (oft verdeckten) Parteinahmen kritisch ins Visier genommen werden.

Linguistische Wortmeldungen sind aber selbst nicht vor fragwürdiger Parteinahme gefeit. Ich werde in diesem Artikel an der Kür zum Unwort des Jahres 2012 exemplarisch vorführen, wie eine fragwürdige Parteinahme entsteht.

Es ist oft nicht leicht $\mathrm{zu}$ erkennen, wann man in einer sprachkritischen Stellungnahme die Grenze von einer wissenschaftlich-,,objektiven“ Position zu einer Parteinahme überschritten hat. Das hat damit zu tun, dass eine moralische Dimension in der Sprachkritik unumgänglich ist. Sie begleitet notwendigerweise alles öffentliche Kommunizieren, unseren Alltag und unser Denken, oft ohne dass wir uns darüber bewusst sind. In Abschnitt 3 werde ich den Framing-Ansatz besprechen, mit dessen Hilfe man verstehen kann, wie solche moralischen Prädispositionen den Diskurs prägen, um den es hier geht. Zunächst soll aber das hier verwendete Konzept der Parteilichkeit vorgestellt werden und wie es sich in sprachkritischen Stellungnahmen niederschlägt.

\section{Parteilichkeit und Parteinahme}

Ein weit verbreitetes laienlinguistisches Vorurteil über Sprache ist die Annahme, dass in ihr Homogenität und Eindeutigkeit herrsche und jede Abweichung davon fehlerhaft sei. ${ }^{1}$ Das Nebeneinander gleichwertiger Formulierungsvarianten, die Mehrdeutigkeit sprachlicher Ausdrücke und die Kontextabhängigkeit dieser Bedeutungen sind demgegenüber der empirische sprachliche Alltag, mit dem wir uns wissenschaftlich auseinandersetzen. Diese Kennzeichen der sprachlichen Realität sind letztlich der Tatsache geschuldet, dass eine Sprache als soziales Konstrukt die Heterogenität und Komplexität einer Gesellschaft und ihrer sozialen Beziehungen widerspiegelt.

Sprache ist nicht nur unser Mittel, um im Diskurs Einverständnis zu erzielen. Wir tragen mit ihrer Hilfe unseren Streit aus, stellen uns selbst und unsere Ansichten mit sprachlichen Mitteln dar, und verfolgen dabei Absichten, die wir nicht immer offenlegen.

Wortbedeutungen kommen im Sprachgebrauch zustande. Da wir es dabei immer mit aus ihrer subjektiven Interessenlage heraus handelnden Personen zu tun haben, darf man nicht davon ausgehen, dass Wortbedeutungen per se neutral sind. In ihnen schlagen sich Haltungen, subjektive Sichtweisen und Parteilichkeiten nieder. Das gilt insbesondere für Ausdrücke, die im öffentlichen Diskurs geprägt werden.

Nicht immer spitzt sich das so zu wie in dem Ausdruckspaar „Kernkraft-Atomkraft“, wo sich Befürworter und Gegner dieser Technologie einst an ihren lexikalischen Präferenzen ablesen ließen. Das Beispiel zeigt aber, dass eine politische Meinung Bestandteil des Lexikoneintrags eines Wortes sein kann. Ein politisch neutrales Lexikon der deutschen Sprache müsste beide Varianten gleichberechtigt nebeneinander gelten lassen. Der Eintrag zum Stichwort „Atomkraftwerk“ im Brockhaus ist vor diesem Hintergrund ein bisschen verräterisch:

1 Dass solche Mythen auch bei der Herausbildung von Standardsprachen eine wichtige Rolle spielen, zeigt Watts (2011) für das Englische. Durrell (2014) deutet an, dass Vergleichbares auch für die deutsche Standardsprache gilt. 
(1) „Atomkraftwerk, umgangssprachl. Bez. für ein mit Kernenergie betriebenes Kraftwerk $(\rightarrow \text { Kernkraftwerk })^{\text {“. }}$.

Das Ausdruckspaar wird hier nicht nur in richtig und falsch aufgeteilt, die Verwendung des Attributs „umgangssprachl.“ versucht darüber hinaus den Ausdruck „Atomkraft“ aus der Standardsprache zu verbannen. Es hat den Anschein, als würde die Auseinandersetzung darüber, welche Ausdrücke zur Standardsprache gehören, mit der Prämisse geführt, dass sich eine Partei mit ihren Ansichten durchsetzen soll, deren Sprachgebrauch sich dann zur Sprachnorm für alle erhebt - getreu dem Motto: die herrschende Sprache (Standardsprache) ist die Sprache der (politisch, ökonomisch) Herrschenden und drückt auch deren Meinung aus.

Das hieße aber dann auch in unserem Fall, dass die ,Minderheitenmeinung ${ }^{6}$ der Atomkraftgegner in der Standardsprache nicht vorkommen dürfte, mithin, wenn man dies verallgemeinert, dass die hier verfolgte Vorstellung von Standardsprache autoritär statt pluralistisch ist. Das widerspräche aber einer zentralen Funktion der Standardsprache für eine Demokratie, nämlich die Meinungs- und Redefreiheit zu ermöglichen, wofür die Möglichkeit, konträre Ansichten zu versprachlichen, essentiell ist.

Anders gesagt, wer für eine demokratische, und deshalb auch pluralistische Gesellschaft eintritt, muss auch für eine vielfältige Sprache eintreten. Es ist offensichtlich so, dass die Variabilität und Pluralität gerade der öffentlichen Sprache gegen autoritäre Versuchungen geschützt werden muss. Linguistische Sprachkritik sollte hier in der Tat wichtige Beiträge liefern können, die aber vorrangig in der Verteidigung vermeintlich abweichenden Sprachgebrauchs bestehen müsste, anstatt den laienlinguistischen Zeigefingerreflex des ,das sagt man nicht“ zu bedienen.

Selbst in dem diesem Anliegen grundsätzlich verpflichteten „Zeitgeschichtlichen Wörterbuch der deutschen Gegenwartssprache“ (Stötzel/Eitz, 2003) findet sich unter dem von Silke Bartsch geschriebenen Eintrag „Atom- vs. Kern-“ die Anmerkung „Aufgrund der häufigen Verwendung des Ausdrucks Atom in der Presse und den übrigen Medien verliert die Vokabel ihren diffamierenden Charakter als Schlagwort." (Bartsch, 2003, 39). Wenn man die Formulierung von Gegnerschaft mit dem Attribut „diffamierend“ abwertet, leistet man sprachlich bereits einer (undemokratischen) Delegitimierung von Opposition Vorschub.

2 Brockhaus: Enzyklopädie in 30 Bänden, 21. völlig neu bearb. Aufl., Band 2: Anau - Ausv, S. 647, Leipzig u.a.: Brockhaus, 2006.

Unter dem Stichwort „Kernkraftwerk“ (Band 14, S. 732) findet sich der Hinweis „,allgemeinsprachlich Atomkraftwerk“. In Band 2 des Brockhaus, S. 645, wird „Atomenergie“ als „häufig verwendete, aber nicht exakte Bez. für die aus den Atomkernen ... gewonnene Energie“ bezeichnet. Insgesamt haben wir es im Brockhaus also mit drei verschiedenen Attribuierungen für den Sprachgebrauch der Atomkraftgegner zu tun: umgangssprachlich, allgemeinsprachlich und „,häufig verwendet, aber nicht exakt“. „Umgangssprachlich“ ist als Kontrast zu standardsprachlich zu verstehen, ,allgemeinsprachlich“ wohl als Kontrast zu „fachsprachlich“. Diese Attribuierungen sind empirisch falsch, da beide Gebrauchsweisen heute gleichermaßen zur Standard-, Umgangs- wie Fachsprache gehören. Sie haben auch ihre parteiliche Konnotation verloren, denn seit Tschernobyl wird die Technologie allgemein kritisch gesehen. Der Brockhaus scheint hier eine längst geschlagene Schlacht weiterführen zu wollen. Dass „Kernkraft“ eine exaktere Bezeichnung als „Atomkraft“ sein soll, erschließt sich überhaupt nicht. Wenn es um begriffliche Präzision ginge, wäre wohl „Atomkernkraft“ ein sehr guter Kandidat. Jedenfalls ist das Morphem „Kern“ wesentlich diffuser in seiner Verwendungsbreite als das Morphem „Atom“. 


\subsection{Ein Beispiel: von Polenz (1966)}

In einem Beitrag zur IDS-Tagung 1966 formulierte Peter von Polenz eine Analyse, die auch heute noch im oben beschriebenen Sinne als beispielhaft gelten kann (von Polenz, 1968). Gegenstand war unter anderem eine Buchbesprechung (im Spiegel 14/1963), in der Hans Magnus Enzensberger den zweiten Band des „Wörterbuch der deutschen Umgangssprache“ von Heinz Küpper vorstellte. Von Polenz griff Enzensbergers Empörung darüber auf, dass die Redewendung „bis zur Vergasung etwas üben (tun)“ zwanzig Jahre nach Auschwitz ,in jedem Vorortzug des Landes“ zu vernehmen sei.

Von Polenz' kritische Replik ist für die hier zu führende Diskussion aus mehreren Gründen interessant. Zunächst einmal äußerte sich in Enzensbergers Einlassung, wie auch von Polenz deutlich machte, die elitäre Haltung eines akademisch Gebildeten, der den eigenen Sprachgebrauch absolut setzt und zum Maßstab für die gesamte Sprachgemeinschaft macht. Von Polenz rügte dabei vor allem die Unterstellung, die Passagiere von Vorortzügen (also, „einfache Leute“) würden den Ausdruck auch so meinen, wie Enzensberger ihn verstand - also als Reminiszenz an Auschwitz.

Eine damals populäre Herleitung des Ausdrucks „bis zur Vergasung“ verortete seinen Ursprung in der Zeit des ersten Weltkriegs mit seinem massiven Giftgaseinsatz. Von Polenz zeigte, dass auch diese Herleitung nicht stimmen kann. Er hatte aber eine Erklärung dafür, warum Germanisten (hier aus den 1920er Jahren) auf diese Idee kamen: die Soldatenzeit war eine der wenigen Möglichkeiten für einen Akademiker jener Zeit, mit „einfachen Leuten“ und ihrer Sprache in Kontakt zu treten. Der tatsächliche Ursprung des Ausdrucks liege eher in der Techniksprache, in der mit Vergasung der Übergang in den gasförmigen Zustand bezeichnet wird. Man denke beispielsweise an den „Vergaser“ als Bestandteil des Automotors.

Die Parteilichkeiten, auf die dieser Beitrag von Polenz' implizit hinweist, sind

(2) a. die Parteilichkeit des demokratisch-humanistischen Wertekonsenses,

b. die des Hörers, der dem Sprecher unterstellt, einen Ausdruck so zu meinen, wie er, der Hörer, ihn (absichtsvoll miss-) versteht,

c. die elitäre Borniertheit des Akademikers, der das eigene Sprachgefühl zum Maßstab für alle macht,

d. eine sprachideologische Positionierung, die sich durch die Hypostasierung sprachlicher Ausdrücke auszeichnet, denen ein Eigenleben zugesprochen wird, das der kultur- und bildungsbewusste Sprachpfleger retten zu müssen meint.

e. $\mathrm{Zu}$ dieser Positionierung gehört eine dekontextualisierte Betrachtung sprachlicher Ausdrücke, die so tut, als könnte man ihre Bedeutung unabhängig davon bestimmen, wer unter welchen Umständen den Ausdruck verwendet.

Eine zeitgemäße linguistische Haltung zu diesen Fragen impliziert aus meiner Sicht wenigstens die folgenden Parteilichkeiten:

(3) a. Die gesamte Sprachgemeinschaft ist als der „Sprachsouverän“ (Hundt, 2009) anzusehen und ihre Rechte sind gegen die Anmaßungen einer Minderheit (hier: der akademischen Schicht) zu verteidigen.

b. Ein Sprachgebrauch, der eine Position außerhalb des demokratisch-humanistischen Wertekonsenses formuliert, ist zu verurteilen.

c. Der Sprecher und seine Absichten sind ins Zentrum zu stellen und alle 
hypostasierenden Überhöhungen der Sprache zu vermeiden (wie beispielsweise die etymologische Begründung der angeblich wahren Bedeutung eines Wortes).

d. Somit ist ganz grundsätzlich einem bildungsbürgerlich-elitären Führungsanspruch in Sachen Sprache entgegenzutreten. Das beinhaltet eine kritische Distanz

gegenüber (selbst ernannten oder dazu berufenen) Sprachautoritäten, und damit auch die Verpflichtung zu einer selbstkritischen (Zurück-)Haltung.

In Enzensbergers Angemessenheitskritik zeigt sich eine Verquickung von Parteilichkeit in eigener Sache und Parteinahmen, die vom Autor nur zum Teil bewusst vollzogen werden. Die begriffliche Unterscheidung dieser beiden Typen von Parteilichkeiten ist für meine Diskussion hier und im Folgenden zentral. In (4) ist angegeben, wie ich diese Begriffe in diesem Text verwende. ${ }^{3}$

(4) Zwei Typen von Parteilichkeiten:

Parteilichkeit in eigener Sache: eine Haltung, die aus der eigenen Perspektive, dem eigenen Interesse heraus bewusst oder unbewusst eingenommen wird

Parteinahme: eine Haltung, bei der man sich bewusst oder unbewusst auf die Seite einer Partei in einem meist öffentlich geführten Streit schlägt

Parteilichkeiten in eigener Sache sind in (2b,c) aufgeführt. (2a,d) und (3a-d) stellen Parteinahmen dar.

\subsection{Parteilichkeiten in linguistischen Wortmeldungen}

Parteilichkeiten sind in öffentlichen Stellungnahmen unvermeidlich und notwendig. Für öffentliche Wortmeldungen aus der Linguistik gilt es vor allem, sich darüber zu verständigen, welche Parteilichkeiten zu vermeiden sind.

LinguistInnen streiten für die eigene Sache. Zu dieser Parteilichkeit sollte man sich bekennen. Schon die Frage, ob die Sprache einer bestimmten Schicht oder sozialen Gruppe als Maßstab für alle gelten soll, zeigt aber, wie schwierig es ist, zu beurteilen, ob eine Parteilichkeit vertretbar ist oder nicht: einerseits ist die Bevorzugung eines Soziolekts gegenüber anderen undemokratisch, also ein Verstoß gegen Grundwerte. Andererseits benötigt beispielsweise der Sprachunterricht wenigstens Vorbilder, an denen die Sprache gelehrt werden soll. Wie soll denn der schulische Deutschunterricht funktionieren, wenn er keine Maßstäbe für die Bewertung der sprachlichen Kompetenz der Schüler verwendet?

Verdächtig macht sich diese Haltung lediglich durch die allzu selbstverständliche Verwendung des akademisch-bildungsbürgerlichen Soziolekts als sprachdidaktischem Maßstab. Die lehrenden und normsetzenden Autoritäten tun dabei ja nichts anderes, als ihre eigene Varietät parteilich gegenüber anderen Varietäten zu privilegieren. Es ist denn auch kein Zufall, dass die sprachliche Bevorzugung des bildungsbürgerlichen Soziolekts in der Schule mit der nachgewiesenen Bevorzugung von Kindern aus gehobenem oder höher gebildetem Elternhaus einhergeht. ${ }^{4}$

3 Der Begriff Parteilichkeit wird hier und im Folgenden als Oberbegriff für Parteilichkeit in eigener Sache und Parteinahme verwendet.

4 Wann hat man schließlich schon einmal davon gehört, dass ein Akademikerkind wegen fehlender Dialektkompetenz eine schlechtere Deutschnote bekommen hätte? 
Parteinahme findet grundsätzlich in jeder sprachkritischen Wortmeldung statt: mit einem bestimmten Sprachgebrauch wird immer auch sein Verwender kritisiert und somit gegen ihn Partei ergriffen. Eine Sprachhandlung ist von der Person, die sie ausführt, nicht zu trennen. Leider wird dieser Aspekt auch in linguistischer Sprachkritik zu wenig beachtet.

Im Rahmen einer linguistischen Angemessenheitskritik werden nicht selten Äußerungen moniert, die in einem öffentlichen Meinungsstreit fallen. Häufig liegt einem Streit der Konflikt zweier inkompatibler, aber moralisch gut begründeter Positionen zugrunde. Sprachkritische Äußerungen werden dann nicht selten zu Parteinahmen, weil ein inkriminierter Ausdruck nur aus der Perspektive einer der Parteien als anstößig empfunden werden kann.

Linguistische Sprachkritik, die so in Auseinandersetzungen eingreift, läuft Gefahr, ungerechtfertigt einer der beiden streitenden Parteien einen moralischen Vorsprung einzuräumen, indem sie quasi mit einem linguistischen Gütesiegel versehen wird.

So unumgänglich es also einerseits ist, Sprachkritik mit einer moralischen Grundlage zu betreiben, so wichtig ist es für linguistische Sprachkritik andererseits, sich nicht in einem Streit instrumentalisieren zu lassen.

\subsection{Unwortkritik als Kritik an Sprechakten}

Die „sprachkritische Aktion Unwort des Jahres“ existiert seit nunmehr über 20 Jahren und kann in Deutschland als die öffentlichkeitswirksamste Aktion von LinguistInnen betrachtet werden. Sie wird als repräsentativ für die Zunft der LinguistInnen in Deutschland wahrgenommen - dass dies völlig unberechtigt ist, ändert nichts an dieser Tatsache.

„Sprachliche Ausdrücke werden dadurch zu Unwörtern, dass sie von Sprechern entweder gedankenlos oder mit kritikwürdigen Intentionen verwendet werden, und dies im öffentlichen Kontext", heißt es auf der Webseite der Aktion, unwortdesjahres.net. Die vier wesentlichen inhaltlichen Kriterien, die genannt werden, sind Verstöße gegen Menschenwürde oder Prinzipien der Demokratie, Diskriminierung und verschleiernder Sprachgebrauch.

Die Jury wählt einmal im Jahr aus eingesandten Vorschlägen aus. Es ist fraglich, ob solche von Laien vorgebrachten Vorschläge tatsächlich differenzieren zwischen einem Ausdruck und den Intentionen, mit denen er verwendet wird, also zwischen dem Wort und dem Sprechakt. In den Jurybegründungen wird meist nicht auf die tatsächlichen kommunikativen Situationen Bezug genommen, in denen ein kritisierter Ausdruck verwendet wurde. Die Absichten der Personen, die ihn verwendeten, bleiben meist im Unklaren. Das kritisierte Wort, so heißt es oft, würde diesen oder jenen Personenkreis diskriminieren - wohlgemerkt, das Wort selbst, nicht die Personen, die das Wort in einer bestimmten Situation verwendeten.

Es wird also keine Sprechaktkritik vorgenommen, sondern eine bloße Wortkritik, die schon aufgrund dieses Umstands hinter dem Stand linguistischen Wissens zurückbleibt. Wenn es richtig ist, dass Wörter erst durch ihre Verwendung ihre konkrete Bedeutung erhalten, dann muss Sprachkritik vor allem Sprechaktkritik sein. Erst, indem wir handeln, können wir gegen moralische Prinzipien verstoßen. Wörter werden erst unmoralisch, wenn sie verwendet werden.

Ich plädiere also dafür, dass die sprachliche Handlung, in der ein Unwort geäußert wird, Gegenstand der Kritik sein sollte und nicht bloß der Ausdruck an sich und was 
man irgendwelchen fiktiven Benutzern dieses Ausdrucks unterstellt. Eine bloß wortfixierte Sprachkritik ist von genau der Art, wie sie von Polenz vor fünfzig Jahren an Enzensberger kritisierte.

Die Unwortjury scheint mir bei ihrer Bewertung vor allem auf die Hörerperspektive zu fokussieren. Unberücksichtigt bleibt dabei, dass ein Ausdruck je nach Interessenlage unterschiedlich verstanden werden kann, dass es auch ein absichtsvolles Missverstehen gibt. Auch die Perspektive des Zuhörers ist alles andere als objektiv und ein Ausblenden der Sprecherabsichten bei der Bewertung birgt das Risiko, unfair zu sein. Wenn es sich nicht gerade um einen offen feindselig-diskriminierenden Ausdruck wie beispielsweise „Pleite-Griechen“ (auf Platz 2 bei der Wahl zum Unwort 2012) handelt, lässt sich der postulierte Unwortcharakter eines Ausdrucks relativ leicht anfechten.

Zur Illustration der Probleme, die sich für die Unwort-Jury durch ihre Vorgehensweise ergeben, möchte ich kurz auf das Unwort des Jahres $2007 \mathrm{zu}$ sprechen kommen. Es lautet „Herdprämie“. Der Ausdruck entstammt der Diskussion um die Initiative zu einem Recht auf einen Betreuungsplatz für über einjährige Kinder und die staatliche Bezuschussung solcher Plätze. Das konservative Lager schlug als Reaktion darauf vor, Familien, die ihre Kinder bis zum Kindergartenalter zuhause lassen möchten, einen Teil des Zuschusses für den dadurch nicht in Anspruch genommenen Betreuungsplatz direkt zukommen zu lassen. Dieser Beitrag wird als „Betreuungsgeld“ bezeichnet. Es kann seit Mitte 2013 beantragt werden.

Im Jahr 2007 wurde lediglich im politischen Diskurs über diesen konservativen Vorschlag gestritten. Seine Realisierung stand noch in weiter Ferne. In der Formulierung „Herdprämie“ drückt sich nach meinem Verständnis eine bekannte feministisch-emanzipatorische Kritik an konservativen Familienvorstellungen aus. Das Betreuungsgeld kann aus dieser Sicht als Vehikel gesehen werden, um die traditionelle patriarchalisch geprägte Einernährerfamilie zu stärken, in der die Frau als bloße Dienstleisterin fungiert und in ihren Entwicklungsmöglichkeiten massiv eingeschränkt ist. Der Herd gilt als Sinnbild dieses angenommenen Unterdrückungsverhältnisses. Folglich ist aus dieser feministischen Sicht an dem Ausdruck nichts anstößig, im Gegenteil, er bringt auf sehr prägnante Weise die Kritik an dem konservativen Vorschlag auf den Punkt. Es ist Kritik an Verhältnissen, nicht an Personen.

Die auf unwortdesjahres.net dokumentierte Jurybegründung fällt recht knapp aus: „Ärgerlich ist insbesondere der Versuch, alle Frauen - darunter auch solche, die der Kindererziehung zuliebe ihre Karriere unterbrechen oder aufgeben - zu «Heimchen am Herd» zu degradieren.“ Seltsam ist hier der Vorwurf der Allquantifizierung (,alle Frauen"), der ohne sachliche Grundlage in den Raum gestellt wird.

Mit dem Ausdruck „Herdprämie“ wurde ja ein konservativ geprägter Vorschlag inklusive seiner Urheber von KritikerInnen als frauenfeindlich beurteilt. Die Unwortjury drehte in ihrer Begründung den Spieß um und stellte die Urheber dieses Ausdrucks nun als frauenfeindlich dar. Selbst, wenn dies nachvollziehbar wäre, so müsste man konstatieren, dass es einer parteilichen Sichtweise bedarf, um die eigentlichen Motive der Urheber des Ausdrucks „Herdprämie“ zu verschweigen, wie dies die Jury tut.

Das Wort wurde im Kontext eines gesellschaftspolitischen Ideenstreits von einer Seite geprägt und es wirkt auch nur aus dem Blickwinkel der anderen (konservativen) Seite tatsächlich anstößig. Die Unwortjury hat also unter dem Deckmantel einer vermeintlich überparteilichen Sprachkritik - ob gewollt oder ungewollt - Partei für die 
konservative Sichtweise ergriffen. Diese verdeckte Parteinahme von der hohen Warte der Wissenschaft aus ist das eigentliche Ärgernis an diesem Vorgang. ${ }^{5}$

Mein persönliches Unwort des Jahres 2009 ist eine Passage aus einem Interview mit Thilo Sarrazin, veröffentlicht in der Zeitschrift Lettre International, Heft 86, S. 197-201, Oktober 2009 (Hervorhebungen von mir):

(5) ,... Integration ist eine Leistung dessen, der sich integriert. Jemanden, der nichts tut, muß ich auch nicht anerkennen. Ich muß niemanden anerkennen, der vom Staat lebt, diesen Staat ablehnt, für die Ausbildung seiner Kinder nicht vernünftig sorgt und ständig neue kleine Kopftuchmädchen produziert. Das gilt für siebzig Prozent der türkischen und für neunzig Prozent der arabischen Bevölkerung in Berlin. ...“

In Unkenntnis dieser Einlassung würde ich mit dem Ausdruck „Kopftuchmädchen“ nur ausgesprochen positive Eigenschaften und Werte assoziieren. Man denkt an das Märchen vom Rotkäppchen und überhaupt kleine Mädchen als Sinnbild von Unschuld und Freundlichkeit. Ein Beschützerinstinkt wird ausgelöst.

Hier wird der Ausdruck „Kopftuchmädchen“ nun im Kontext einer vor Gehässigkeit, Verachtung und Rassismus triefenden Tirade verwendet und mit dieser krass negativen Konnotation aufgeladen. Eine extremere Umwertung eines positiven Ausdrucks wird man lange suchen müssen. Aber es sind eben der Kontext und die niederen Beweggründe des Autors, die diese negative Umwertung auslösen. Der Ausdruck „Kopftuchmädchen“ an sich ist völlig harmlos. Wenn es sich dabei also um ein Unwort handelt, dann ist es eindeutig ein Un-Sprechakt, der sich hier gerade nicht, wie bei der Unwortkür üblich, in einer offensichtlich verwerflichen Wortprägung zeigt, sondern in der negativen Aufladung eines eigentlich positiven Ausdrucks.

\section{Framing}

Ein theoretischer Ansatz, der die Subjektivität und Perspektivität kommunikativen Handelns zu analysieren erlaubt, ist die Framingtheorie. In mehreren Arbeiten stellte George Lakoff seine framing-basierte Analyse der politischen Kommunikation und insbesondere der Propagandasprache der konservativen Partei der USA vor (Lakoff, 1996, 2002, 2004, 2008; Lakoff/Wehling, 2008). Die darin entwickelten Kategorien sind für das Verständnis von Parteilichkeit ausgesprochen nützlich, weshalb ich den Ansatz im Folgenden kurz diskutieren möchte.

Essentielle Bestandteile von Lakoffs Theorie sind einerseits die von Charles Fillmore begründete Frame-Semantik, andererseits Lakoffs eigener Ansatz einer erfahrungsbasierten Kognitionstheorie, die neben Frames auf Metaphern (Lakoff/Johnson, 1980) und Narrativen als zentralen Bestandteilen der menschlichen Kognition aufbaut. Lakoff knüpft an die von Goffman (1974) entwickelte Theorie des Framings an. Es ist eine Theorie über die kognitive Verankerung menschlicher Erfahrung. Die Grundidee ist, dass wir Episoden und Situationen, die wir erleben, auf

5 Es bleibt noch festzuhalten, dass der Ausdruck „Betreuungsgeld“ aus der beschriebenen emanzipatorisch-feministischen Perspektive einen Euphemismus darstellt, der genauso sprachkritisch zu beanstanden wäre, ginge man so vor wie die Jury. Aus der hier von mir vertretenen Position ergibt sich für diesen Fall, dass die Unwortjury antidemokratisch agierte und somit gegen ihre eigenen Prinzipien verstieß. Solche Ausdrücke, die legitime Meinungen prägnant zum Ausdruck bringen, sind aus sprachkritischer Sicht zu verteidigen. 
der Grundlage unserer bisherigen Erfahrungen interpretieren (framen). Dies geschieht unwillkürlich, meist unbewusst, und es ist unausweichlich. Mithilfe von Framing konstruieren wir aus einem Strom unverbundener Beobachtungen und Vorkommnisse Situationen und Ereignisse.

Eine fruchtbare Weiterentwicklung erfuhr der Framing-Ansatz in der Analyse der Medien und der öffentlichen Kommunikation. Studien zum Agenda Setting politischer Parteien und Organisationen und zur Realitätskonstruktion durch Nachrichten in den Massenmedien stehen hierbei im Zentrum (siehe dazu beispielsweise Scheufele, 1999).

Ein Frame ist ein Netzwerk von Wissens- und Erfahrungsbestandteilen. Das Wissen über die Struktur einer Fahrt in einem öffentlichen Verkehrsmittel, ihre Voraussetzungen und typischen Begleitumstände, kann man zum Beispiel als einen solchen Frame repräsentieren. Frames sind also ein allgemeines Modell der Wissensrepräsentation. Sie werden durch Erlebnisse, Beobachtungen oder einen sprachlichen Ausdruck ,evoziert“. Die davon inspirierte Frame-Semantik ist eine Theorie der Wortbedeutung, die die ansonsten übliche Trennung von enzyklopädischem Wissen und Wortbedeutung aufgibt. Wichtig ist auch der assoziative Charakter des Modells: ein Frame kann eine ganze Reihe anderer Frames evozieren.

Nicht nur Erfahrung und sprachliche Bedeutung, sondern Denken selbst ist nach Lakoff in dieser Weise organisiert. Das gilt auch für die anderen Bestandteile dieser Theorie, Metaphern und Narrative, die als spezielle Arten von Frames gesehen werden können. Zum metaphorischen Charakter des Denkens gehört, dass wir Neues oder Spezielles in der Form vertrauter Muster kategorisieren und charakterisieren. So entstehen metaphorische Übertragungen wie beispielsweise ,gut ist oben - schlecht ist unten“ für die Dimension der Moral oder die Konzeptualisierung eines Gefallens als geschäftliche Transaktion („Ich schulde dir was.“, „Wie kann ich dir das zurückzahlen? ") usw.

Narrative sind einfache Erzählschemata, anhand derer wir zusammenhängende Handlungen und Vorgänge kategorisieren und bewerten. Zu Narrativen gehört immer auch eine moralische Dimension. Mit ihrer Hilfe bewerten wir Verhalten als positiv oder negativ. Wie in einem kleinen Theaterstück sind in einem Narrativ Rollen vergeben: die des (guten) Helden, aus dessen Perspektive erzählt wird, die des (bösen) Kontrahenten, des Opfers, der Helfer usw.

Narrative sind wichtig für die öffentliche Kommunikation. Fast immer, wenn Ereignisse in den Medien dargestellt werden, werden sie in die Form eines Narrativs gebracht. In der politischen Auseinandersetzung finden sie häufig Verwendung. George W. Bush beispielsweise hätte, wie Lakoff ausführt, aufgrund der Dürftigkeit seiner bisherigen Leistungen kaum eine Chance auf das Amt des US-Präsidenten gehabt. Geholfen hatte ihm der offene Umgang mit seinem bewältigten Alkoholismusproblem. Dadurch wurde das Läuterungs-Narrativ abgerufen. Es machte in der Öffentlichkeit aus einem vermeintlichen beruflichen Versager eine Respektsperson.

Lakoff beschreibt Narrative als sehr tiefsitzende kognitive Schemata, die an gleichfalls sehr tiefsitzende moralische Prinzipien anknüpfen. Sie helfen uns, zwischen Gut und Böse, richtigem und falschem Verhalten zu unterscheiden. Das heißt nicht, dass sie archaisch sein müssen - wie die aus Märchen bekannten Erzählmuster. Der Feminismus zum Beispiel habe unserer Kultur eine Reihe moderner Narrative hinzugefügt. Frames, Narrative und Metaphern sind zwar kognitiv verankert, sie 
wurzeln aber letztlich, so Lakoff, in der Kultur einer Gemeinschaft und sind insofern nicht notwendigerweise allgemeinmenschlich.

Für das Verständnis von Lakoffs Framing-Theorie ist nun das Folgende wichtig: Es ist nicht so, dass wir mithilfe von Frames Erlebtes quasi nachträglich kategorisieren. Vielmehr ist es umgekehrt so, dass Frames unsere Wahrnehmung und unser Denken leiten. Das geht so weit, dass Frames darüber bestimmen, welche Fakten wir zur Kenntnis nehmen - nämlich solche, die zu dem Frame passen, den wir gerade verwenden - und welche nicht. Frames sind nicht alternativlos und die Frames, die wir mental abgespeichert haben, können einander widersprechen. Wir sind auch in der Lage, bei der Wahrnehmung desselben Vorgangs zwischen Frames zu wechseln vorausgesetzt, uns stehen dafür alternative Frames zur Verfügung. Aber je nachdem, welchen Frame wir verwenden, wird unsere Wahrnehmung bestimmte Aspekte der Realität unterdrücken und andere hervorheben.

Lakoffs Hauptanliegen in diesen Arbeiten ist eine Analyse des kommunikativen Erfolgs der Republikanischen Partei in den USA mit dem Zweck, die politische Kommunikation der Demokratischen Partei zu verbessern. Der wichtigste Ratschlag ist dabei, und das gilt wohl generell für öffentliche Kommunikation, dass man sich nicht auf das Framing seines Kontrahenten einlassen darf, sondern versuchen muss, dem ein eigenes Framing entgegenzusetzen. Alleine mit dem Appell an die Vernunft und der Hoffnung, dass die Fakten für sich selbst sprechen, schafft man keine Überzeugungen. Das eigene Framing wirkt dabei umso überzeugender, wenn es an im allgemeinen Bewusstsein tief verankerte positive Werte anknüpft und insofern auch die emotionale Dimension anspricht.

Oft ist man geneigt, gerade nicht so vorzugehen, sondern sprachlich an den Setzungen seines Kontrahenten anzuknüpfen, um ihnen zu widersprechen. Damit ist man aber bereits in die Falle gegangen. Richard Nixon konnte die amerikanische Öffentlichkeit mit der Äußerung „I'm not a crook“ (,,ich bin kein Betrüger“) nicht überzeugen, im Gegenteil.

Lakoff illustriert dies in seinen Texten gerne mit der Anweisung „Denken Sie nicht an einen Elefanten! “, die zu befolgen eine psychologische Unmöglichkeit darstellt. Die sprachliche Negation funktioniert psychologisch nicht so, wie man sich das vielleicht vom Standpunkt der Logik aus vorstellen könnte - also als eine Art mentales Radiergummi, das das Negierte mental zum Verschwinden bringt. Um den Satz verstehen zu können, muss man das Wort „Elefant“" interpretieren und damit evoziert man zwangsläufig die Vorstellung eines Elefanten. Wer also eine Idee unterdrücken möchte, sollte sie ignorieren und an ihre Stelle alternative eigene Ideen setzen, und sie in einer eigenen Sprache formulieren. Man widerspricht einem Gedanken am Besten, indem man ihn durch einen eigenen verdrängt. Die Rolle von Perspektiven und Deutungsrahmen bei der Beurteilung öffentlichen Sprachgebrauchs wurde spätestens mit dem Semantikstreit und der Diskussion um das Begriffe besetzen in den 70er und 80er Jahren zum Bestandteil der linguistischen Analyse der politischen Sprache in Deutschland. Für politische Kommunikation ist das Durchsetzen der eigenen Perspektive essentiell. Ein Sachverhalt wird erst durch seine Deutung, sein Framing, zu einer relevanten Nachricht. Ein unparteiisches Framing ist aber eigentlich nicht möglich. 


\section{4 „Opfer-Abo“ - das Unwort des Jahres 2012}

Die unabhängige Jury „Unwort des Jahres“ kürte am 15.1.2013 den Ausdruck „OpferAbo“ zum Unwort des Jahres 2012. ${ }^{6}$ Der Vorgang kann als besonders anschauliches Beispiel für die Wirkmächtigkeit von Framing und Parteilichkeiten gelten. Die Parteilichkeit in der Jurybegründung ist anhand einer ganzen Fülle unlauterer rhetorischer Mittel erkennbar, wie ich im Folgenden anhand einer Detailanalyse zeigen werde.

\subsection{Der Beleg - das Kachelmanns-Interview im Spiegel 41/2012 vom 8.10.2012}

Anlässlich des Erscheinens ihres gemeinsamen Buches (Kachel-mann/Kachel-mann, 2012) veröffentlichte der Spiegel ein längeres Interview mit dem Ehepaar Kachelmann. Es trägt den Titel „Kollektiver Blutrausch“, der sich insbesondere auf die öffentliche Vorverurteilung bezieht, der Jörg Kachelmann ausgesetzt war. Gegenstand des Buches ist die Aufarbeitung der Affäre um Jörg Kachelmann aus Sicht des Ehepaars.

Zur Erinnerung: Jörg Kachelmann, beliebter Fernsehmoderator und Mit-Eigentümer einer Firma, die Dienstleistungen rund um das Wetter und Wettervorhersagen erbringt, wurde unter großem Medienaufsehen aufgrund eines Vergewaltigungsvorwurfs von der Staatsanwaltschaft Mannheim angeklagt. Er verbrachte 132 Tage in Untersuchungshaft, aus der er kurz vor Verhandlungsbeginn aufgrund einer Entscheidung des Oberlandesgerichts Karlsruhe (gegen das Votum der Mannheimer Justiz) entlassen wurde.

Der Freispruch für Kachelmann am 31.5.2011 war eigentlich vorhersehbar. Die Zweifel an der Glaubwürdigkeit des vermeintlichen Opfers, die im Laufe der Verhandlung deutlich wurden, waren für eine Verurteilung zu groß. Dass die Staatsanwaltschaft das Verfahren trotz der ihr bekannten Schwächen in den Aussagen der Belastungszeugin eröffnete und öffentlich führte, wird nicht nur vom Ehepaar Kachelmann so gedeutet, dass man ihr unbedingt glauben wollte. ${ }^{7}$ Die Opfer-AboTheorie - wenn wir es so nennen wollen - ist auch der Versuch, für das Verhalten der Mannheimer Justiz eine Erklärung zu finden.

Das Verfahren wurde seitens der Boulevardpresse mit einer beispiellosen Kampagne begleitet, die einer Vorverurteilung Kachelmanns gleichkam, aber auch für Frau D. und andere Beteiligte zu einer erheblichen Belastung wurde. ${ }^{8}$

Thomas Knellwolfs Fazit: „Jörg Kachelmann und vielleicht auch die Frau, die ihn angezeigt hat, werden Getriebene bleiben. Die anonymen Diffamierungen in Kampfblogs im Internet, in deutscher Sprache in dieser Dimension unbekannt, werden

6 Die m.E. etwas unglückliche Schreibweise mit Bindestrich statt „Opferabo“ wird hier aus der Originalquelle, dem Spiegel, übernommen.

7 Eine sehr ausführliche und ausgesprochen sachliche Darstellung des Falles in all seinen Aspekten verfasste der Schweizer Journalist Thomas Knellwolf (Knellwolf, 2011).

8 Seriösere Medien wie die Zeit und auch der Spiegel zeigten eher eine Parteinahme pro Kachelmann. Knellwolf (2011, 253): „Deutsche Leitmedien, darunter die angesehensten und meistgekauften Zeitungen und Zeitschriften der Republik, begleiteten den Fall vielfach einseitig, manchmal faktenarm, immer meinungsstark und überraschend willfährig. Die Berichterstattung verkam allzu oft zur Selbstdarstellung. Die traditionellen Medien kämpften um die Wahrheit, statt dass sie versuchten, die Realität abzubilden und zu hinterfragen.“ 
weiter existieren, die Verschwörungsgeschichten nicht vergessen.“ (Knellwolf, 2011, 253)

Für den künftigen Umgang mit der Kausa gibt der Richter in der Urteilsbegründung eine dringliche Empfehlung:

(6) „Bedenken Sie, wenn Sie künftig über den Fall reden oder berichten, dass Herr Kachelmann möglicherweise die Tat nicht begangen hat und deshalb zu Unrecht als Rechtsbrecher vor Gericht stand. Bedenken Sie aber auch umgekehrt, dass Frau (Name gestrichen) möglicherweise Opfer einer schweren Straftat war. Versuchen Sie, sich künftig weniger von Emotionen leiten zu lassen. Unterstellen Sie die jeweils günstigste Variante für Herrn Kachelmann und Frau (Name gestrichen) und führen Sie sich dann vor Augen, was beide möglicherweise durchlitten haben. Nur dann haben Sie den Grundsatz ,in dubio pro reo" verstanden."

Die hier formulierte Forderung, zwei einander widersprechende Framings gleichzeitig gelten zu lassen, kann als eine allgemeine Forderung an den öffentlichen Umgang mit Streitfällen aller Art in einer Demokratie gelten. Ich halte sie auch für einen essentiellen Maßstab für gute Sprachkritik.

Im Vordergrund des Spiegel-Interviews steht die Sichtweise der Kachelmanns, derzufolge Jörg Kachelmann Opfer einer Verleumdung durch Frau D. sei, die aber vor allem deshalb ihre Wirkung habe entfalten können, weil Frau D. die Justiz und wichtige Teile der Medienöffentlichkeit auf ihrer Seite gehabt habe. An keiner Stelle werden pauschal Vorwürfe gegen Frauen im Allgemeinen formuliert, oder gar Vergewaltigungsopfer verhöhnt. Einige Passagen enthalten allerdings pauschale negative Aussagen über Institutionen der Justiz und Opferhilfeorganisationen.

Im Folgenden nun die Passage, die zu der kritisierten Verwendung des Ausdrucks „Opfer-Abo“ führte:

SPIEGEL: Sie nennen in Ihrem Buch fast alle Akteure, von denen Sie sich verfolgt fühlten, beim vollen Namen - auch das vermeintliche Vergewaltigungsopfer Claudia D.

Miriam Kachelmann: Das Wort Opfer ist im Zusammenhang mit ihr komplett fehl am Platze. Sie hat nach Jörgs Freispruch der „Bunten“ inklusive beeindruckender Fotostrecke für mutmaßlich viel Geld ein großes Interview gegeben. Nicht zuletzt hat auch „Emma“ sie bei vollem Namen genannt.

SPIEGEL: Claudia D. war über viele Jahre eine Ihrer Freundinnen, Herr Kachelmann. Wenn man mal davon ausgeht, dass die Vergewaltigung wirklich nicht stattfand, fragt man sich bis heute: Wie konnten Sie in dieser Frau einen solchen Hass provozieren?

Miriam Kachelmann: Wenn ich da mal einhaken darf: Ich finde schon die Frage falsch. Was habe ich in jemandem ausgelöst, dass derjenige eine kriminelle Handlung begeht, in diesem Fall eine Falschbeschuldigung? Wenn umgekehrt ein Mann einer Frau mit einer Keule auf den Kopf haut,

9 Aus dem Wortlaut der Urteilsverkündung, zitiert nach der Frankfurter Allgemeinen Zeitung, 31.5.2011, http://www.faz.net/aktuell/gesellschaft/kriminalitaet/wortlaut-der-freispruch-fuer-joergkachelmann-1639748.html. 
wird die Frau ja auch nicht aufgefordert, darüber nachzudenken, was sie vielleicht vorher in dem Mann ausgelöst hat, oder?

SPIEGEL: Es ist doch sinnvoll, sich und sein Tun mitunter zu hinterfragen.

Miriam Kachelmann: Natürlich. Aber man muss doch die Verhältnismäßigkeit sehen. Diese Frau hat versucht, Jörg mit falschen Beschuldigungen zu vernichten.

Jörg Kachelmann: Das ist das Opfer-Abo, das Frauen haben. Frauen sind immer Opfer, selbst wenn sie Täterinnen wurden. Menschen können aber auch genuin böse sein, auch wenn sie weiblich sind. (Der Spiegel 41/2012, S.

138)

Die inkriminierte Äußerung steht im Kontext einer Provokation durch den Interviewer, Spiegel-Redakteur Thomas Tuma, auf die zunächst Miriam Kachelmann reagiert - und zwar interessanterweise, indem sie ein zugrundeliegendes Framing explizit macht und ein alternatives Framing an dessen Stelle zu setzen versucht. Da der Interviewer nicht locker lässt, verdeutlicht Jörg Kachelmann noch einmal die gemeinsame Sichtweise des Ehepaars, hier fällt dann der Ausdruck „Opfer-Abo“. Es ist eindeutig, dass die Haltung des Interviewers Gegenstand der Kritik ist, auch weil sie den Kachelmanns exemplarisch für eine weit verbreitete Haltung erscheint. Es ist der Vorwurf einer naiven Haltung, nach der Frauen nie böse Absichten verfolgen würden, sondern höchstens (von Männern) dazu getrieben würden, und so selbst als Täterinnen noch Opferstatus erhielten.

In dem Interview wird im Grunde die Existenz eines wohlbekannten überkommenen Geschlechterklischees beklagt, das uns in verschiedener Gestalt immer wieder begegnet: Frauen sind schwach, potentielle Opfer, empathisch, passiv, und Männer sind stark, potentielle Täter, unsensibel, aktiv usw. Wenn nun, wie die Kachelmanns im konkreten Fall, man den Eindruck hat, die Entscheidungen der Justiz beruhten eher auf solchen Stereotypen als auf dem Bemühen um eine möglichst objektive Einzelfallbetrachtung, dann sollte dies auch öffentlich thematisiert werden können.

Die Schlussfolgerung der Kachelmanns, dass dadurch Frauen, die sich an jemandem rächen möchten, eine sehr wirkungsvolle Waffe in die Hand bekommen, ist keine pauschale Anklage gegenüber dem weiblichen Geschlecht, da ja nicht gesagt wird, dass jede Frau rachsüchtig ist. Deshalb wird auch nicht pauschal jede Frau, die eine Vergewaltigung anzeigt, unter Verleumdungsverdacht gestellt. Von Ermittlungsbehörden und Gerichten wird lediglich verlangt, beide Möglichkeiten gleichermaßen in Betracht zu ziehen und die angezeigten Fälle so objektiv wie möglich zu beurteilen. ${ }^{10}$ Die Sorge der von den Kachelmanns kritisierten Institutionen gilt in erster Linie der vergleichsweise niedrigen Anzeigenquote bei Vergewaltigungen. Man möchte Frauen, die vergewaltigt wurden, nicht durch $\mathrm{zu}$ offensive Verfolgung von Falschbeschuldigerinnen verschrecken. ${ }^{11}$ Diese Praxis kann natürlich die Gefahr einer

10 Die im Jahr 2013 bekannt gewordenen Fälle von Gustl Mollath und Horst Arnold können als Beleg dafür gelten, dass vor manchem deutschen Gericht tatsächlich bei Gewaltdelikten weiblichen Opferzeuginnen eher geglaubt wird als männlichen Angeklagten und dadurch Justizirrtümer mit existentiellen Konsequenzen für die unschuldig Verurteilten zustande kommen.

11 „Doch auch wenn Falschbeschuldiger vor Gericht scheitern und der Angeklagte freigesprochen wird - eine Strafverfolgung müssen sie nicht fürchten. Wegen Vortäuschens einer Straftat wird laut Polizeistatistik pro Jahr nur gegen 0,2 Prozent der Verdächtigen ermittelt. Dahinter steckt der Wille der Staatsanwälte, potenzielle Anzeigeerstatter nicht abzuschrecken. Die Strafjustiz lebt von 
geschlechtsbezogenen Diskriminierung von Männern mit existenziellen Folgen in sich bergen. Diese Sorge auszusprechen, ist in keiner Weise moralisch fragwürdig.

Kritisiert wird durch die Unwortjury, dieser Eindruck drängt sich auf, eher die mit dem Ausdruck „Opfer-Abo“ bekundete Meinung als der Ausdruck selbst. Anstößig wirkt der Ausdruck in der Öffentlichkeit vor allem deshalb, weil die Realität dahinter bestritten wird, oder der nicht nur von den Kachelmanns geführte Diskurs um eine mögliche Benachteiligung von Männern vor Gericht einfach unbekannt ist. Wie soll er aber bekannt werden, wenn er nicht in eine prägnante sprachliche Form verpackt werden darf? Es ist also nicht wirklich das reine Sprachgefühl, das dazu führt, dass man an dem Ausdruck Anstoß nimmt, sondern das Sprachgefühl rückgekoppelt mit einem Common Sense, den die Kachelmanns ja gerade auf Grund vermeintlicher blinder Flecken problematisieren, und zwar mit ehrenwerten Motiven. Irritierend wirkt auch, dass für die Jury offenbar überhaupt keine Rolle gespielt hat, wer das gerügte Unwort artikulierte, und mit welcher Intention. Wenn sich ein Politiker oder ein Boulevardjournalist eines Unworts bedient, dann spielt für die Bewertung auch eine Rolle, dass es sich um wohlkalkulierte Äußerungen im Dienste kommerzieller oder politischer Interessen handelt. Wer aber auch nur den kleinsten Funken Empathie für das Rufmordopfer (durch die Boulevardpresse) und mögliche Verleumdungsopfer Kachelmann aufbringt, müsste dessen Wortwahl eigentlich mit Nachsicht behandeln. Empörte bedienen sich eben selten einer Knigge-gerechten Sprache.

\subsection{Die Begründung der Jury}

In diesem Abschnitt will ich mich detailliert mit der Begründung der Unwort-Jury auseinandersetzen. Sie sei im Folgenden zunächst im Zusammenhang dokumentiert, Satznummerierungen wurden von mir in eckigen Klammern hinzugefügt, um auf die einzelnen Sätze referieren zu können:

„Begründung: [1] Im Herbst 2012 sprach Jörg Kachelmann in mehreren Interviews (z.B. im Spiegel vom 8.10.2012) davon, dass Frauen in unserer Gesellschaft ein „Opfer-Abo“ hätten. [2] Mit ihm könnten sie ihre Interessen in Form von Falschbeschuldigungen - unter anderem der Vergewaltigung gegenüber Männern durchsetzen. [3] Das Wort „Opfer-Abo“ stellt in diesem Zusammenhang Frauen pauschal und in inakzeptabler Weise unter den Verdacht, sexuelle Gewalt zu erfinden und somit selbst Täterinnen zu sein. [4] Das hält die Jury angesichts des dramatischen Tatbestands, dass nur 5-8 \% der von sexueller Gewalt betroffenen Frauen tatsächlich die Polizei einschalten und dass es dabei nur bei 3-4\% der Fällen [sic! ] zu einer Anzeige und einem Gerichtsverfahren kommt,[Fußnotenverweis auf eine aktuelle Statistik des Bundesministeriums für Familie, Senioren, Frauen und Jugend, R.V.] für sachlich grob unangemessen. [5] Das Wort verstößt damit nicht zuletzt auch gegen die Menschenwürde der tatsächlichen Opfer. [6] Die Jury urteilt damit in keiner Weise darüber, ob und inwiefern Einzelpersonen von Verleumdungen betroffen sein können und somit auch nicht über den Fall Kachelmann. [7] Sie kritisiert vielmehr einen Wortgebrauch, der gängige Vorurteile in Bezug auf eine Vortäuschung von Vergewaltigungen oder eine

Hinweisen aus dem Volke, in Werbekampagnen wird dazu ermuntert, Straftaten bei der Polizei zu melden. Es wäre also kontraproduktiv, die Hinterbringer selbst in Bedrängnis zu bringen.“ (Rückert, 2011) 
Mitschuld der Frauen bestätigt. [8] Ausdrücke dieser Art drohen letztlich den zivilgesellschaftlichen und juristischen Umgang mit sexueller Gewalt in bedenklicher Weise zu beeinflussen. ${ }^{\text {"12 }}$

Die ersten beiden Sätze enthalten bereits einige bemerkenswerte rhetorische Auffälligkeiten. Erstens: In Kenntnis der publizistischen Aktivitäten des Ehepaars Kachelmann fragt man sich, warum hier Jörg Kachelmann alleine erwähnt wird und seine Ehefrau, die sich in diesem Diskurs so sehr engagierte wie er, unterschlagen wird. Das in der Begründung als einziger Beleg angeführte Spiegel-Interview wurde schließlich mit beiden geführt und das Studium der Textstellen zeigt, dass es sich um ihre gemeinsame und gemeinsam formulierte Position handelt. Jörg Kachelmann twitterte im Anschluss an die Kür auch prompt, dass seine Frau eigentlich den Ausdruck „Opfer-Abo“ erfunden habe. In einem anderen Tweet meldet sie sich auch selbst zu Wort: „Miriam Kachelmann, die mutmassliche Urheberin des „Unwort des Jahres“: ,Wer unser Buch gelesen hat, weiß, dass wir den Begriff Opfer-Abo zu Recht verwenden. Natürlich gibt es Frauen, die sexuelle Gewalt erfinden und natürlich treffen sie auf eine Gesellschaft, die diesen Umstand unerträglich und jede Kritik daran politisch unkorrekt findet. Die Wahl zum Un-Wort des Jahres bestätigt somit eindrucksvoll die tatsächliche Existenz des Opfer-Abos. “"13

Macht dies einen Unterschied? Ja, einen bedeutenden. Jörg Kachelmann, im Zuge der Prozessberichterstattung in einem Teil der Presse nahezu dämonisiert, wurde zwar vom Gericht freigesprochen, aber damit ist die Verknüpfung des Namens mit dem Vergewaltigungs-Topos noch lange nicht aus dem öffentlichen Gedächtnis getilgt. Jede künftige Assoziation seines Namens mit dem Wort „Vergewaltigung“, so wie auch hier geschehen (um im folgenden Satz [2] die „Vergewaltigung“ unterzubringen, wird extra ein Einschub gemacht), verstetigt somit auch die Diffamierung. Wie gesagt, die eigentlich korrekte Erwähnung des Ehepaars Kachelmann hätte dies vermieden. Sie hätte aber auch den hier mit aufgerufenen Topos des Geschlechterkonflikts konterkariert. Es passt ja nicht in das Bild der unterstellten sprachlichen Frauenverachtung, wenn sie von einer Frau kommt. Der Einschub ,-- unter anderem der Vergewaltigung -“" in Satz [2] hätte, um der diffamierenden Assoziation vorzubeugen, zwingend von dem Zusatz begleitet werden müssen, dass Jörg Kachelmann vom Vorwurf der Vergewaltigung freigesprochen wurde und somit seine Unschuld erwiesen ist. Am Besten hätte man ihn aber weggelassen.

Zweitens: Von welchen Frauen ist in den ersten beiden Sätzen die Rede? Satz [1] verwendet den indefiniten Plural, der hier generisch zu verstehen ist: Frauen allgemein haben also ein Opfer-Abo. Gehen wir zu Satz [2] über. Das Pronomen „sie“ referiert auf die erwähnten Frauen. Wie ist das Pronomen zu interpretieren? Eigentlich so wie „Frauen“ im ersten Satz, also generisch. Es ist von allen Frauen die Rede.

Was wird nun von Jörg Kachelmann nach Lesart der Jury über alle Frauen gesagt? Um das verstehen zu können, müssen wir uns mit dem Verb „könnten“ beschäftigen. Grundsätzlich kann der hier verwendete Konjunktiv zwei Funktionen haben: er ist zum Einen ein Mittel der indirekten Redewiedergabe. Dies ist hier sehr wahrscheinlich.

12 Aus der Presseerklärung der Unwortjury vom 15.1.2013, zitiert nach der im Internet unter http://www.unwortdesjahres.net/fileadmin/unwort/download/ pressemitteilung_unwort2012.pdf veröffentlichten Fassung, zuletzt abgerufen am 24.10.2014.

13 Tweet vom 15.1.2013 unter http://www.twitlonger.com/show/knevgd. 
Eigentlich ist der Konjunktiv I dafür die bessere Wahl, aber „können“ wäre formgleich mit dem Indikativ und in diesem Fall ist es üblich, auf den Konjunktiv II zu wechseln. Er ist aber auch ein Mittel, um den Irrealis auszudrücken, also Dinge, die - prinzipiell möglich, aber nicht real sind. Unter dieser Interpretation erschienen aber die in [3] gemachten Vorwürfe völlig überzogen. Offenbar ist dies nicht gemeint.

Das Modalverb „können“ ist nun selbst mehrdeutig in seiner Verwendung. Es drückt zunächst eine Fähigkeit aus wie in „Ich kann schwimmen“. In dieser Bedeutung ist nichts darüber gesagt, ob jemand auch die Absicht hat, etwas zu tun (hier: ob ich die Absicht habe, zu schwimmen). In einer zweiten Bedeutung geht es um Erlaubnis oder Ermöglichung. Hier ist eine Absicht aber meist per Implikatur mitgedacht: „Ich habe meine Mutter gefragt: Ich kann schwimmen.“ Die Erwähnung, dass einem eine Handlung erlaubt ist, ist im Allgemeinen nur dann von Relevanz, wenn man auch beabsichtigt, diese Handlung durchzuführen. Diese Implikatur schwingt auch in Satz [2] mit. So gelangen wir zur folgenden Paraphrase für Satz [2]:

(7),,Alle Frauen hätten die Absicht, ihre Interessen gegenüber Männern in Form von Falschbeschuldigungen - unter anderem der Vergewaltigung - durchzusetzen. Das würde ihnen durch das Opfer-Abo auch ermöglicht."

Nur in dieser aberwitzigen Interpretation machen die darauffolgenden Vorwürfe in der Jury-Begründung Sinn. Entspricht das aber auch dem, was die Kachelmanns gesagt haben? Natürlich nicht. Schauen wir uns dazu, drittens, die Wendung „Interessen durchsetzen“" an, die in Satz [2] zu finden ist. Wie in dem Spiegel-Interview (siehe oben) dargelegt, geht es den Kachelmanns tatsächlich um Frauen, die kriminell werden. Eine Frau, die einen Mann fälschlicherweise einer Straftat bezichtigt, um sich an ihm zu rächen, ihm das Sorgerecht für das gemeinsame Kind vorzuenthalten oder sich berufliche Vorteile zu verschaffen (das sind die im Spiegel-Interview erwähnten Fälle), ist also in der Paraphrase der Unwort-Jury einfach nur eine Frau, die ihre Interessen durchsetzt. Eines der Kriterien für die Auswahl zum Unwort des Jahres ist eine euphemistische Sprache. Die Jury hat sich an dieser Stelle ganz offensichtlich selbst dieses unschönen Mittels bedient.

Wollte man Satz [2] so umformulieren, dass er die Intention der Kachelmanns wiedergibt, müsste er eher so lauten:

(8),,Es gebe böswillige Frauen, die versuchten, mittels einer Falschbeschuldigung Männern zu schaden, um sich an ihnen zu rächen, ihnen das Sorgerecht für das gemeinsame Kind vorzuenthalten oder sich berufliche Vorteile zu verschaffen. Das Opfer-Abo mache es diesen Frauen leicht, Fehlurteile in ihrem Sinne vor Gericht zu erwirken.“

Wichtig ist mir hier der Unterschied zwischen der Allquantifizierung „Alle Frauen“ in (7) und der Existenzquantifizierung „Es gebe böswillige Frauen“ in (8). Der Vorwurf der pauschalen Verdächtigung von Frauen gründet wesentlich auf der fälschlicherweise unterstellten Allquantifizierung. Hier noch einmal die Liste der in den Sätzen [1] und [2] verwendeten sprachlichen Mittel, um den Ausdruck „Opfer-Abo“ mit Eigenschaften aufzuladen, die er in seiner ursprünglichen Verwendung gar nicht hat. 
1. Falsche Referenz: Jörg statt Jörg und Miriam Kachelmann

2. Diffamierendes Framing: Aufruf des Vergewaltigungsframes, und damit verbunden Bekräftigung der öffentlichen Diffamierung Jörg Kachelmanns

3. Verschweigen von Entlastendem: Jörg Kachelmann ist unschuldig

4. Irreführendes Framing: Geschlechterkonflikt

5. Falsche Referenz: Frauen allgemein statt böswillige Frauen

6. Falsche Bedeutung: Erlaubnis- statt Möglichkeitsinterpretation von „können“, dadurch Unterstellung, dass alle Frauen als böse betrachtet würden

7. Euphemismus: Interessen statt böswillige Absichten

Für zwei nicht sehr lange Sätze ist das schon eine ziemliche Häufung sprachlicher Beanstandungen. Lesen wir weiter. In Satz [3] fällt der Satzteil ,in diesem Zusammenhang" auf. Es geht hier ja schließlich um einen von der Unwort-Jury in Satz [2] kreierten Zusammenhang, der ursprünglich gar nicht existierte und den die Kachelmanns auch nicht meinten. Aber natürlich trifft der Vorwurf in [3] auf die Paraphrase in (7) zu. Auch das ist ein bekannter rhetorischer Trick, das absichtsvolle Missverstehen: man unterstellt seinem Kontrahenten eine völlig unseriöse Äußerung, die er gar nicht gemacht hat, einen Strohmann, und prügelt dann höchst empört darauf ein. Verfehlung Nr. 8:

\section{Strohmann-Manöver}

Das Manöver setzt sich in den Sätzen [4] und [5] fort. Sie dienen auch der Anknüpfung an die Jurykriterien und bilden insofern den Kern der Begründung (,sachlich grob unangemessen“, „Menschenwürde“). Das Aufrufen von Zahlen aus Studien ist leicht als rhetorische Strategie der Anrufung einer höheren Autorität zu verstehen, mit der man die eigene Haltung zu immunisieren versucht. Nur haben diese Zahlen, so schlimm sie sind, und auch von den Kachelmanns in besagtem Interview beklagt werden, nichts mit dem Anliegen der Kachelmanns zu tun.

Diesen Punkt zu verstehen, ist für das, worum es mir mit dieser Analyse geht, wichtig. Es erscheint fast unmöglich, bei Konflikten wie dem zwischen Herrn Kachelmann und Frau D. eine neutrale Position einzunehmen. Entweder sie hat Recht und er ist ein Monster, dessen Gefährlichkeit im Grunde durch den Freispruch noch gesteigert wird, weil er zeigt, wie raffiniert das Monster vorgeht. Oder er hat Recht und sie hat es geschafft, mit Lügen und Manipulationen seinen Ruf zu ruinieren und wenn er nicht so viel Geld in Gutachter gesteckt hätte, hätte ihn die aus seiner Sicht inkompetente Mannheimer Justiz („Opfer-Abo“) ins Gefängnis gesteckt und seine Existenz zerstört.

Aus seiner Perspektive ist die Straftat, die hier verhandelt wird, eine Verleumdung. Aus ihrer Perspektive ist es eine Vergewaltigung. Genau eine dieser beiden Straftaten hat tatsächlich stattgefunden; wir wissen aber nicht, welche. Der oben zitierte Richterspruch hat auf das Dilemma hingewiesen, das sich hieraus ergibt.

Wenn man Jörg Kachelmann zitiert, sollte man dies auch aus seiner Perspektive tun, 
sonst kann man ihn ja gar nicht verstehen. Und in dieser Perspektive hat der Verweis auf Studien zur Dunkelziffer von Vergewaltigungen überhaupt keinen Sinn, weil es den behaupteten Zusammenhang nicht gibt. Jörg Kachelmann hat mit Vergewaltigung so viel $\mathrm{zu}$ tun wie jeder andere unbescholtene Bürger. Das ist der Kern der Unschuldsvermutung, die hier zwingend anzuwenden ist.

Um ihm dies zu unterstellen, muss man seine Äußerung aus ihrem ursprünglichen Kontext reißen und in einen passenden anderen Kontext stellen. ${ }^{14}$ Auf diese Weise kann man aber aus jeder gut gemeinten, aber provokanten Äußerung ein Unwort machen.

\section{Irreführende Kontextualisierung}

Ein Beispiel: „Gute Mädchen kommen in den Himmel, böse überall hin: Warum Bravsein uns nicht weiterbringt" ist der Titel eines viel gelesenen Frauenratgebers von Ute Erhardt. Im Kontext der Äußerungen der Kachelmanns klingt dieser emanzipatorisch gemeinte Titel allerdings wie die Aufforderung zu einer Straftat.

Die Rede von den „tatsächlichen Opfern“" in Satz [5] blendet die Opfer von Verleumdung und Rufmord aus. Gegen deren Menschenwürde verstößt die Jury durch diese Missachtung tatsächlich:

10. Moralische Verfehlung: Missachtung der Opfer von Verleumdungen

Das rhetorische Manöver, das mit den Sätzen [6] bis [8] vorgeführt wird, dient einerseits der Absicherung gegen den Vorwurf, auf das Anliegen der Kachelmanns nicht eingegangen $\mathrm{zu}$ sein. Gleichzeitig sollen von Verleumdungstaten aber nur „Einzelpersonen“ betroffen sein. Hier wird bagatellisiert. Von der Dunkelziffer der Verleumdungsopfer ist gleich gar nicht die Rede.

\section{Bagatellisierung einer Straftat: Verleumdungen sind Einzelfälle}

Die Bagatellisierung wird auch durch das folgende rhetorische Mittel vorbereitet: während das quantitative Ausmaß von Vergewaltigungen so groß ist, dass man es nur in Form von Zahlen darstellen kann, sind von Verleumdungen bloß „Einzelpersonen“ betroffen. Die Implikatur, die auf diese Weise entsteht, stiftet einen Zusammenhang zwischen der Häufigkeit einer Straftat und ihrer Verfolgungswürdigkeit. Folgt man dieser Logik, dann müsste sich die Polizei auf die Verfolgung von Falschparkern konzentrieren und die Aufklärung von Morden, die ja zahlenmäßig kaum ins Gewicht fallen, sein lassen. Weitere (ungewollte) Implikaturen entstehen dadurch, dass Verleumdung und Vergewaltigung als Alternativen dargestellt werden, so als ob die Verfolgung einer Verleumdung durch die Justiz die Verfolgung einer Vergewaltigung ausschlösse.

Vor Gericht ist aber jeder verhandelte Fall ein Einzelfall und jeder Justizirrtum ist ein Justizirrtum $\mathrm{zu}$ viel. Alles andere wäre eine unmoralische Position. Das zahlenmäßige Aufrechnen verschiedener Straftaten, wie es die Jury hier betreibt, ist

14 Das ist nun obendrein genau der Kontext, in den Jörg Kachelmann durch die vermeintliche Verleumdung gestellt wurde. Die Jury setzt also in gewisser Weise diese Verleumdung fort - aus Kachelmanns Sicht. 
unserer Rechtsvorstellung zurecht fremd. Obendrein steht zu befürchten, dass die Justiz Straftaten, die selten vorkommen, auch leichter übersieht. Gerade in Bezug auf seltene Straftaten ist es deshalb womöglich hilfreich, die Wachsamkeit der Strafverfolgungsbehörden anzumahnen.

Über die tatsächlichen Zahlen an Falschbeschuldigungen haben die Kachelmanns und die von ihnen und der Zeit-Autorin Sabine Rückert zitierten Experten ${ }^{15}$ obendrein eine ganz andere Auffassung. Das wird hier bewusst unterschlagen - nach dem, was wir in Abschnitt 3 über Framing gelernt haben, ist es allerdings nicht mehr erstaunlich.

\section{Verschweigen von Gegenargumenten: das tatsächliche Ausmaß von Falschbeschuldigungen ist umstritten}

Es wird damit eindeutig für die von den Kachelmanns kritisierten Institutionen Partei ergriffen. Die Jury bemüht sich nicht um Neutralität in einem Meinungsstreit, in dem zwei moralisch gut begründbare Positionen im Konflikt stehen. Im Gegenteil, sie beteiligt sich an der Tabuisierung einer kontroversen, aber möglicherweise wichtigen Meinungsäußerung. ${ }^{16}$ Das ist eine undemokratische Haltung.

\section{Aufgabe der Neutralität und Tabuisierung einer legitimen Meinung}

15 , ,Die in Statistiken meist verfälschte, tatsächlich aber relativ hohe Falschaussagenquote gerade in Sexualstrafverfahren', schreibt der Richter am Bundesgerichtshof, Ralf Eschelbach, in seinem Kommentar, ,wird nicht ausreichend beachtet." Stattdessen werde den zumeist weiblichen Opferzeugen ein unangebrachter moralischer Kredit eingeräumt.

Dabei ist die Problematik in Staatsanwaltschaften, bei Rechtsmedizinern und Aussagepsychologen durchaus bekannt. Der Kieler Psychologieprofessor Günter Köhnken, einer der gefragtesten Glaubwürdigkeitssachverständigen Deutschlands, schätzt die Quote der Falschbeschuldiger unter den von ihm Untersuchten auf 30 bis 40 Prozent. Klaus Püschel, Direktor des Rechtsmedizinischen Instituts Hamburg, das die größte deutsche Opferambulanz betreibt, konstatiert, im Jahr 2009 hätten sich 27 Prozent der angeblich Vergewaltigten bei der ärztlichen Untersuchung als Scheinopfer erwiesen, die sich ihre Verletzungen selbst zugefügt hatten. Nur in 33 Prozent der Fälle habe es sich erwiesenermaßen um echte Opfer gehandelt, bei den restlichen 40 Prozent sei die Rechtsmedizin zu keinem eindeutigen Ergebnis gekommen. Die Tendenz zum Fake hat - laut Püschel - erst in den vergangenen Jahren eingesetzt. Bis dahin habe die Falschbeschuldigungsrate über Jahrzehnte konstant bei fünf bis zehn Prozent gelegen.“ (Rückert, 2011)

Der hier zitierte Klaus Püschel wirkte auch als Gutachter im Kachelmann-Verfahren mit. Seine Angaben zum Anteil von Falschbeschuldigungen werden auch von Miriam Kachelmann zitiert (Kachel-mann/Kachel-mann, 2012, 334).

16 In der Welt vom 31.10.2012 wird unter der Überschrift „Fall Kachelmann - Nun ist Claudia D. die Beklagte“ (http://www.welt.de/vermischtes/weltgeschehen/ article110460692/Fall-KachelmannNun-ist-Claudia-D-die-Beklagte.html) über die Eröffnung eines - inzwischen abschlägig beschiedenen - Zivilprozesses berichtet, bei dem Jörg Kachelmann Forderungen auf Schadensersatz gegen Claudia D. erhob. In der Kommentarspalte zum Artikel auf der Webseite der Welt äußerte sich im Oktober 2012 eine „Tanja“ folgendermaßen: „Ich wünsche Frau D[...] von Herzen alles Gute! Was Kachelmann und co von sich geben ist fatal für die Position von Vergewaltigungsopfern vor Gericht. Insbesondere die Kreation von Worten wie „Opferindustrie“ und „Opfer-Abo“ beweist doch m.e. die üble Intention. Diese Wortkreationen schlage ich bei der Wahl des „Unwortes des Jahres“ vor! “ Nach Angaben der Unwort-Jury wurde „Opfer-Abo“ nur einmal vorgeschlagen. Falls die hier zitierte Tanja die Vorschlagende war, dann bestätigt die zitierte Wortmeldung die Parteinahme des Vorschlags. Diese ist deshalb fatal, weil es sich um Parteinahme in einem anhängigen Zivilprozess handelte. Die Jury hat sich also möglicherweise instrumentalisieren lassen. 
Interessant an Satz [7] ist überdies, dass man zur Verstärkung der Argumentation einen weiteren Aspekt ins Spiel bringt, der nicht einmal mit dem Ausdruck „Opfer-Abo“ in Zusammenhang zu bringen ist, nämlich das Vorurteil von der „Mitschuld der Frauen“ an ihrer Vergewaltigung. Das ist auch deshalb so perfide, weil der Ausgangspunkt für die Opfer-Abo-Äußerung im Spiegel-Interview ja gerade die Frage nach Kachelmanns Mitschuld an seiner vermeintlichen Verleumdung war. Ein Nebelkerzen-Manöver, das alles auf den Kopf stellt.

\section{Nebelkerze: Verwirrung stiften durch irrelevante Zusatzargumente}

Der in [7] verwendete Ausdruck von den ,gängigen Vorurteilen in Bezug auf eine Vortäuschung von Vergewaltigungen" wiederum ist deshalb unangebracht und tendenziös, weil man eben nicht im selben Atemzug das - ich behaupte das jetzt einfach mal - genauso gängige Vorurteil, dass jeder Mann ein potenzieller Vergewaltiger sei, erwähnt. Über angeblich gängige Vorurteile lässt sich im Übrigen leicht schwadronieren. Es fragt einen ja ohnehin keiner, ob man dafür Belege hat. Was ist die Bedeutung von ,gängig“ und werden Personen, die verschieden Partei ergreifen, dasselbe für gängig halten?

15.Verwendung weder beweis-, noch widerlegbarer Behauptungen

Satz [8] schwingt dann wieder die große Moralkeule. Dass es der Jury gelungen sein könnte, mit ihrer Kür tatsächlich den zivilgesellschaftlichen und juristischen Umgang mit Verleumdungen in bedenklicher Weise zu beeinflussen, dürfte ihr kaum in den Sinn gekommen sein. Zur Sicherheit noch einmal: Satz [8] ist deshalb unangebracht, weil die in Satz [3] auf Grundlage der falschen Rede-Wiedergabe in [1] und [2] vorgenommene Kontextualisierung falsch ist. Sie ist nicht nur in dem Sinne falsch, dass die Sprecherintention falsch wiedergegeben wurde. Sie ist auch in dem Sinne falsch, dass man den Ausdruck „Opfer-Abo“ erst einmal in den Vergewaltigungskontext bringen muss, um ihm die behaupteten negativen Konnotationen anheften zu können. Und dies hatte erst die Jury getan. Schließlich gibt es ja nur den „Spiegel“-Beleg, in dem der Kontext ein anderer ist. Die dem Ehepaar Kachelmann durch Satz [8] ungerechtfertigt unterstellte Absicht ist im Übrigen geradezu ehrenrührig.

Mein Unwort des Jahres 2013, eigentlich natürlich Un-Sprechakt, ist deshalb die Kür der Unwortjury zum Unwort des Jahres 2012. Auf der Webseite der Aktion „Unwort des Jahres“ werden vier Kriterien genannt, die für die Wahl einer Äußerung zum Unwort des Jahres ausschlaggebend sein können. Die Begründung der UnwortJury verstößt nach meiner Analyse tatsächlich gegen alle vier Kriterien: Verstoß gegen das Prinzip der Menschenwürde (Diffamierung eines Rufmord-Opfers, Verharmlosung von Verleumdung); Verstoß gegen Prinzipien der Demokratie (Tabuisierung einer legitimen Minderheitenmeinung); Diskriminierung einzelner gesellschaftlicher Gruppen (Männer, insbesondere männliche Verleumdungsopfer); euphemistische, verschleiernde oder irreführende Sprache (,Interessen durchsetzen“ und die meisten anderen benannten rhetorischen Fehlleistungen). 
Ich bin weit davon entfernt, den Mitgliedern der Jury einen unmenschlichen Geist zu unterstellen, im Gegenteil. Im Übrigen sollte man die doch eher populistische Veranstaltung der Unwort-Kür auch nicht allzu ernst nehmen. Worüber sich die UnwortJury aber im Klaren sein sollte - mehr als sie es zu sein scheint -, ist, dass ihre UnwortKür selbst Teil der öffentlichen Kommunikation ist und nicht etwa über ihr steht. Sie sendet nicht nur das von ihr beabsichtigte Signal für mehr sprachliche Sensibilität. Sie kritisiert tatsächlich auch nicht Wörter, sondern kommunikative Handlungen konkreter Personen und Institutionen, die sie damit auch an den Pranger stellt. Hier hat sie den Falschen getroffen.

Ein Ziel meiner Erörterungen ist es, zu zeigen, dass eine der Jury-Position entgegengesetzte Haltung genauso oder gar noch plausibler begründet werden kann. Der besprochene Fall bietet sich einfach an, um das Phänomen der Koexistenz zweier völlig inkompatibler Perspektiven auf dieselbe Sache zu thematisieren, und die Rolle, die der Streit zwischen solchen Perspektivierungen in der öffentlichen Kommunikation spielt. Denn es ist häufig so, dass eine Perspektive den öffentlichen Diskurs dominiert und andere Perspektiven unterdrückt - das kann gerade aufgrund der Inkompatibilität auch nicht anders sein. Das Ringen um diese „Lufthoheit über den Stammtischen“ ist geradezu das Wesen öffentlicher Kommunikation. Das Besondere am Anliegen der Kachelmanns ist, dass sie eine solche Lufthoheit direkt thematisieren und $\mathrm{zu}$ skandalisieren versuchen. Dies möchte ich im folgenden Abschnitt noch etwas genauer beleuchten.

\subsection{Opfer-Abo-Framing}

Es dürfte aus dem Vorgesagten klar geworden sein, dass bei der Unwortkür von 2012 eine Parteinahme vorliegt. Für diese Parteinahme kann sich die Jury aber weder auf die Sprachwissenschaft, noch auf die von ihr selbst genannten Kriterien berufen.

Die Annahme ist naheliegend, dass die Jurymitglieder dem Anliegen der Kachelmanns eher ablehnend gegenüberstehen - zumindest muss man die JuryBegründung so interpretieren. Mit anderen Worten: die Jurymitglieder handeln im Einklang mit ihrer persönlichen Einschätzung in der Sache, von der sie wohl fälschlicherweise - aber auch typischerweise - glauben, dass es die einzig vernünftige, richtige, objektive Position ist. Diese Art von Irrtum, das Verwechseln der eigenen Perspektive (und des Common Sense) mit Objektivität, ist typisches Merkmal eines besonders gelungenen Framings.

Jörg Kachelmann wurde durch die monatelange mediale Kampagne um sein Gerichtsverfahren im öffentlichen Bewusstsein mit der Rolle des Frauenverächters belegt. Er hatte parallel zahlreiche Beziehungen mit Frauen gepflegt, die voneinander nichts wussten und die er permanent über sein Leben belog. Eine von ihnen, Frau D., brachte ihn mit einer Vergewaltigungsanzeige vor Gericht. All dies wurde in der Presse ausführlich ausgeschlachtet.

Der Freispruch, quasi die Negation des Vergewaltigungsvorwurfs, kann dieses Framing nicht rückgängig machen. Wenn Jörg Kachelmann sich nun über Frauen äußert, dann ist für jemanden, der unter dem Eindruck des Common Sense steht, schon aufgrund dieses Framings die Erwartung hoch, dass Kachelmann sich abfällig geäußert haben muss, nach dem Motto: „Ich weiß zwar nicht, was das Wort bedeuten soll, aber wenn es Kachelmann gesagt hat, dann muss es Frauen gegenüber abfällig gemeint sein.“ 
Die Begründung der Unwort-Jury und viele zustimmende Reaktionen belegen sehr anschaulich die Wirkmächtigkeit eines solchen Framings.

Narrative haben in Lakoffs Theorie der öffentlichen Kommunikation eine wesentliche Bedeutung. Sie strukturieren die Art und Weise, wie komplexe Geschehnisse medial präsentiert werden und weisen insbesondere moralisch aufgeladene Rollen an die Personen zu, um die es geht. Der Fall Kachelmann ruft eine ganze Reihe von Narrativen zum Geschlechterverhältnis auf, unter anderen:

- Don Juan I: der reiche, berühmte Mann, der seine Stellung zum eigenen Vorteil ausnutzt, keine Grenzen mehr kennt, bis hin zu gewalttätigem Verhalten;

- Don Juan II: der Mann, der keiner Frau widerstehen kann und sich so selbst in Schwierigkeiten bringt;

- der Aufsteiger, dessen Hochmut vor seinem Fall kommt;

- die Frau, die sich ihren Märchenprinz angelt und sich in seinem Ruhm sonnt, dann prompt von der Wirklichkeit aus ihren Träumen gerissen wird;

- die geltungssüchtige Egoistin, die sich um den Lohn ihrer Gefügigkeit betrogen sieht;

- die viel zu lange schweigende Frau, die die Erniedrigung durch ihren Partner nicht mehr hinnimmt und sich wehrt.

Einige dieser Narrative sind vom feministisch inspirierten Diskurs um die Geschlechtergleichstellung geprägt. Andere Narrative entspringen dem traditionellen Bild des Geschlechterverhältnisses, wie es insbesondere in der Regenbogenpresse gepflegt wird. Dieses Konglomerat aus emanzipatorisch-feministischen und BoulevardNarrativen führt zu einem Framing (in einem Teil der Öffentlichkeit), in dem die Opferund Täterrollen nach Geschlecht vergeben werden - verkörpert in geradezu paradigmatischer Weise durch Alice Schwarzers Prozesskommentierung in der BILD.

Abweichungen von dieser Rollenzuteilung fallen durch das Wahrnehmungsraster Männer, die von Frauen missbraucht oder verleumdet werden, werden dadurch buchstäblich unsichtbar. Abwehrreaktionen, wie wir sie beispielsweise durch Opferschutzverbände zu hören bekommen (siehe auch Satz [8] der Jury-Begründung), dienen auch der Behauptung dieses Framings gegen eine widersprechende Realität. Es ist aber ein Framing, das keine andere Perspektive als die der Frau duldet, wo es eigentlich moralisch angezeigt wäre, die Perspektive des Opfers, und zwar beider möglicher Opfer, einzunehmen.

Wer sich im Einklang mit einem öffentlich geteilten Framing wähnt, muss auch weniger damit rechnen, dass seine Worte kritisch abgewogen werden. Die oben herausgearbeiteten rhetorischen Fehlgriffe in der Jurybegründung sind ein gutes Beispiel für eine damit einhergehende sprachliche Nachlässigkeit. Die Begründung scheint mir eher ein Appell zu sein, dem Ausdruck „Opfer-Abo“ ein bestimmtes Framing zu verpassen. Es ist dafür nur wichtig, dass die richtigen Schlüsselbegriffe fallen. Den Rest kann man sich dann denken. Hat man das Framing nachvollzogen, ist die genaue Argumentation eigentlich nebensächlich.

Die Begründung liest sich so auch als der absurde Vorwurf an Jörg Kachelmann, 
sich nicht an das von ihm und seiner Frau mit dem Ausdruck „Opfer-Abo“ kritisierte Framing zu halten. Jörg Kachelmann wird so erneut Opfer des öffentlichen Images, das ihm verpasst wurde. Diese Mechanismen sind zur Genüge bekannt. Eine sprachkritische Jury sollte über sie eigentlich aufklären, anstatt selbst auf sie hereinzufallen.

Hat man verstanden, wie Framing funktioniert, wird aber auch klar, dass die Kachelmanns eine kontraproduktive kommunikative Strategie wählten: wer die Benachteiligung von Männern thematisieren möchte, sollte dies nicht dadurch tun, dass er die Bevorzugung von Frauen beklagt - auch wenn dies zwei Seiten derselben Medaille sind. Die Kachelmanns sprechen allerdings auch von einer ,männermordenden Justiz", und schreiben auch in ihrem Buch natürlich vor allem aus der Perspektive eines männlichen Verleumdungsopfers. Es ist bezeichnend, dass dieses mit dem Common Sense inkompatible Framing völlig durch das Raster der öffentlichen Aufmerksamkeit zu fallen scheint, während die Formulierung vom Opfer-Abo für Frauen auf solche Resonanz trifft.

Was wäre geschehen, wenn Jörg Kachelmann gesagt hätte: „Das ist das Täter-Abo, das Männer haben. Männer sind immer Täter, selbst wenn sie Opfer wurden. Menschen können aber auch genuin gut sein, auch wenn sie männlich sind"?

Sehr wahrscheinlich wäre diese Formulierung gar nicht beachtet, oder mit einem erstaunten ,ja aber, das bestreitet doch niemand!“ abgetan worden. Dies ist das Dilemma, in dem man sich befindet, wenn man eine Position vertritt, die konträr zum Common Sense verläuft: wenn man an das bestehende Framing anknüpft, wird man missverstanden, und wenn man ein eigenes Framing verwendet, wird es ignoriert.

George Lakoff hat genau dieses kommunikative Dilemma für die Demokratische Partei im politischen Diskurs der USA herausgearbeitet. Sein Ratschlag ist klar: der Fehler ist nicht das alternative Framing (also „Männer haben ein Täterabo“), im Gegenteil. Es muss aber wieder und wieder in den Diskurs eingespeist werden, um Spuren zu hinterlassen und Wirkung zu entfalten.

\section{Fazit}

Als Ergebnis meiner kritischen Analyse der Initiative „Unwort des Jahres“ als der populärsten öffentlichen Wortmeldung aus Reihen der Linguistik in Deutschland plädiere ich dafür, dass linguistische Sprachkritik das Folgende berücksichtigen soll:

1. Da es in öffentlichen Auseinandersetzungen keine objektive Positionierung gibt, sollten die Parteilichkeiten einer Wortmeldung offengelegt werden. Parteinahmen, die über den allgemein anerkannten Wertekanon hinausgehen, sollten unterbleiben.

2. Der anerkannte Wertekanon für Sprachkritik, wie er sich unter anderem in den Kriterien der Unwortinitiative ausdrückt, ist zu ergänzen um das Kriterium, dass sprachliche Vielfalt zu bewahren ist und Minderheiten nicht in ihrem Recht, ihre Sichtweise auszudrücken, beeinträchtigt werden dürfen.

3. Linguistische Sprachkritik stellt einen Sprechakt und seine Rezeption in den Mittelpunkt. Sprachliche Ausdrücke sind im Kontext der Situation, in der sie verwendet werden, zu bewerten. Sprecher und Hörer können gleichermaßen an der Produktion eines Unworts beteiligt sein.

Oft macht erst die dekontextualisierte Wiederverwendung durch andere aus einem 
Wort ein Unwort. Wenn diese Dekontextualisierung erst durch die Jury vorgenommen wird, wie im Falle des Unworts 2012, dann ist es eigentlich auch die Jury, die aus einer provokanten, aber im Kontext angemessenen Wortwahl ein Unwort macht - erst recht, wenn sie obendrein dem Wort eine andere als die gemeinte Deutung verpasst.

Die Kür zum Unwort des Jahres wird als eine von SprachwissenschaftlerInnen durchgeführte sprachkritische Aktion wahrgenommen und fällt so auch unter die in der Aachener Erklärung angemahnten Bemühungen um öffentliche Wortmeldungen der Sprachwissenschaft. Wenn sich die Jury aber, wie in der FAZ vom 23.1.2013 geschehen, von einem klassischen Philologen (Gerrit Kloss) zurecht vorhalten lassen muss, philologisch ungenau gearbeitet zu haben, ${ }^{17}$ dann müssen sich die Jurymitglieder mehrheitlich auch Unterzeichner der Aachener Erklärung -, vorhalten lassen, ihrer Sache und ihrer Zunft einen Bärendienst erwiesen $\mathrm{zu}$ haben. Sprachbewertungen werden nicht nur auf der Grundlage von Prinzipien vorgenommen, sondern auch vor dem Hintergrund eines Common Sense, der auf in der Öffentlichkeit momentan vorherrschenden Framings beruht, durch die unbewusst Parteinahmen übernommen werden. Sprachkritik hat dann Legitimationsprobleme, wenn der kritisierte Ausdruck lediglich nicht im Einklang mit dem Common Sense steht: die Ächtung einer legitimen Minderheitenmeinung ist undemokratisch, die Ächtung einer inhumanen Minderheitenmeinung ist legitim. Bei der Unwortkür 2012 war Ersteres der Fall. Sie resultierte aus Voreingenommenheit und Vorverurteilung.

Wir beobachten inzwischen die Unwortkür als eine Art Modeerscheinung. So kürt die Düsseldorfer Börse ein Börsenunwort und die nationale Armutskonferenz führt eine eigene Liste von sozialen Unwörtern. Die Parteilichkeiten sind in diesen Fällen offensichtlich. Öffentliche Stellungnahmen aus der Sprachwissenschaft beanspruchen eine andere sprachkritische Autorität. Man muss das Unwortküren aber nicht gleich sein lassen. Es würde genügen, wenn linguistische Sprachkritik selbstkritisch Parteilichkeiten reflektiert und auf der Höhe ihrer Möglichkeiten operiert.

Die vornehmste Aufgabe für linguistische Sprachkritik, das sei hier noch einmal betont, besteht aber darin, die Freiheit des sprachlichen Ausdrucks und den sprachlichen Variantenreichtum gegen autoritäre Versuchungen zu verteidigen. Die Linguistik sollte sich nur im Ausnahmefall selbst an solchen autoritären Handlungen beteiligen.

\section{Literatur}

Bär, Jochen A. und Thomas Niehr (2012). Alternativen zum Elfenbeinturm. Die Linguistik will stärker in die Öffentlichkeit hineinwirken. Aptum 8.3: 281-287.

Bartsch, Silke (2003). Atom- vs. Kern-. In: Stötzel/Eitz (2003), 33-39.

Durrell, Martin (2014). Mit der Sprache ging es immer schon bergab. In: Plewnia, Albrecht / Witt,

Andreas (Hg.), Sprachverfall? Dynamik - Wandel - Variation. Reihe:Jahrbuch des Instituts für Deutsche Sprache 2013.Berlin: de Gruyter Mouton. Seite11-32.

Goffman, Erving (1974).Frame Analysis: An Essay on the Organization of Experience.New York: Harper \& Row.

Markus Hundt (2009).Normverletzungen und neue Normen. In: Konopka, Marek / Strecker, Bruno (Hrsg.), Deutsche Grammatik - Regeln, Normen, Sprachgebrauch. Berlin: de Gruyter, 117-140.

Kachelmann, Jörg und Miriam Kachelmann (2012). Recht und Gerechtigkeit. Ein Märchen aus der Provinz. München: Wilhelm Heyne Verlag.

17 Einige Details meiner oben vorgetragenen Kritik an der Jury-Begründung sind auch in Gerrit Kloss' Kommentar bereits benannt worden. Seiner Auffassung, dass die Jury bloß philologisch schlecht gearbeitet habe, aber in der Sache genügend Hinweise für eine frauenfeindliche Einstellung Jörg Kachelmanns vorlägen - nur in anderen Textstellen -, habe ich hier widersprochen. 
Knellwolf, Thomas (2011).Die Akte Kachelmann. Anatomie eines Skandals. Zürich: Orell Füssli Verlag.

Lakoff, George (1996).Moral Politics: What Conservatives Know That Liberals Don't.University of Chicago Press, 1996.

Lakoff, George (2002).Moral Politics: How Liberals and Conservatives Think.University of Chicago Press, 2002. Weitgehend unveränderte, mit einem neuen Nachwort versehene 2. Auflage von Lakoff (1996).

Lakoff, George (2004).Don't think of an elephant! Know Your values and frame the debate. The essential guide for progressives. White River Junction, Vermont: Chelsea Green Publishing. Lakoff, George (2008). The political mind. A cognitive scientists guide to your brain and its politics.New York: Penguin Books.

Lakoff, George und Mark Johnson (1980).Metaphors we live by. Chicago u.a.: University of Chicago Press.

Lakoff, George und Elisabeth Wehling (2008). Auf leisen Sohlen ins Gehirn. Politische Sprache und ihre heimliche Macht. Heidelberg: Carl-Auer Verlag.

von Polenz, Peter (1968). Sprachkritik und sprachwissenschaftliche Methodik. In Trier, Jost u.a. (Hrsg.), Sprachnorm, Sprachpflege, Sprachkritik. Jahrbuch 1966/67 des Instituts für deutsche Sprache, 159-184.

Rückert, Sabine (2007). Unrecht im Namen des Volkes. Ein Justizirrtum und seine Folgen.

Hamburg: Hoffmann und Campe Verlag.

Rückert, Sabine (2011). Lügen, die man gerne glaubt. Die ZEIT Nr. 28 vom 7.7.2011. Url: http://www.zeit.de/2011/28/DOS-Justiz/

Scheufele, Dietram A. (1999). Framing as a Theory of Media Effects. Journal of Communication 49:103-122.

Stötzel, Georg und Thorsten Eitz (2003).Zeitgseschichtliches Wörterbuch der deutschen Gegenwartssprache. 2., erweiterte und aktualisierte Auflage. Hildesheim u.a.: Georg Olms Verlag. Watts, Richard J. (2011).Language Myths and the history of English. Oxford: Oxford University Press.

Prof. Dr. Ralf Vogel

Universität Bielefeld

Fakultät für Linguistik und Literaturwissenschaft

Postfach 100131

D-33501 Bielefeld

mail: ralf.vogel@uni-bielefeld.de

http://www.uni-bielefeld.de/lili/personen/rvogel/ 